\title{
Meningococcal Endotoxin in Lethal Septic Shock Plasma Studied by Gas Chromatography, Mass-Spectrometry, Ultracentrifugation, and Electron Microscopy
}

\author{
Petter Brandtzaeg, ${ }^{*}$ Klaus Bryn," Peter Kierulf," Reidun Øvstebo," Ellen Namork," Brit Aase, ${ }^{\ddagger}$ and Erik Jantzen \\ Departments of *Pediatrics and ${ }^{\S}$ Clinical Chemistry, Ullevål University Hospital, and Departments for ${ }^{\star}$ Vaccines and of ${ }^{*}$ Immunology, \\ National Institute of Public Health, Oslo, Norway
}

\begin{abstract}
We have compared gas chromatography and mass spectrometry (GC-MS) analysis with the Limulus amebocyte lysate (LAL) assay to quantify native meningococcal lipopolysaccharides (LPS) in five patient plasmas containing $>5 \mu \mathrm{g} /$ liter by LAL. 3-Hydroxy lauric acid (3-OH-12:0) was used as a specific lipid A marker of neisserial LPS. The quantitative LAL results were confirmed by GC-MS $(r=0.98, P=0.006)$. Seven patient plasmas were centrifuged at $103,000 \mathrm{~g}$ and the sedimentation behavior of native LPS compared with reference plasma proteins and with apo $\mathrm{A1}$ and apo B100 representing high and low density lipoproteins. After 15 min of centrifugation, $84 \pm 2 \%$ (mean \pm SE) of the recovered LPS were found in the lower onethird of the centrifuged volume, whereas $6 \pm 1 \%$ remained in the upper one-third volume, indicating that meningococcal endotoxin circulates as complexes with high sedimentation coefficients. Bacterial outer membrane fragments were detected in the bottom fractions of three patient plasmas examined by means of electron microscopy. In three patient plasmas ultracentrifuged for $60 \mathrm{~min}$ at $103,000 \mathrm{~g}$, the levels of apo $\mathrm{A1}$ and apo $B 100$ revealed minor changes, whereas only $1 \pm 1 \%$ of the recovered LPS remained in the upper one-third and $91 \pm 2 \%$ were found in the lower one-third volume. Few bioreactive LPS appear to be complexed with high and low density lipoproteins in meningococcal septic shock plasma. (J. Clin. Invest. 1992. 89:816-823.) Key words: apolipoproteins A1 and B100 • Limulus test $\bullet$ Neisseria meningitidis
\end{abstract}

\section{Introduction}

Bacterial lipopolysaccharides (LPS) elicit a complex pattern of inflammatory reactions when introduced into the circulation of mammals $(1,2)$. Previous studies of patients with systemic meningococcal disease have demonstrated an association between the levels of LPS, as measured with the chromogenic Limulus amebocyte lysate (LAL) ${ }^{1}$ assay upon hospital admis-

Address reprint requests to Dr. Brandtzaeg, Department of Pediatrics, Ullevål University Hospital, N-0407 Oslo 4, Norway.

Received for publication 9 May 1990 and in revised form 22 October 1991.

1. Abbreviations used in this paper: EU, endotoxin unit; GC-MS, gas chromatography/mass spectrometry; LAL, Limulus amebocyte lysate; 3-OH-10:0, 3-hydroxy decanoic acid; 3-OH-12:0, 3-hydroxy lauric acid; 3-OH-14:0, 3-hydroxy myristic acid.

J. Clin. Invest.

(C) The American Society for Clinical Investigation, Inc.

0021-9738/92/03/0816/08 \$2.00

Volume 89, March 1992, 816-823 sion and the development of severe septic shock, renal failure, adult respiratory distress syndrome, and fatal outcome (3). Furthermore, the levels of LPS at admission have been quantitatively reflected in a multitude of parameters that are assumed to play a significant role in the development of multiple organ failure: notably tumor necrosis factor- $\alpha$; interleukin 1 and interleukin 6; the activation state of the complement, kallekreinkinin, coagulation, and fibrinolytic systems; polymorphonuclear granulocyte activation products; and the circulating levels of vasoactive intestinal polypeptide (4-10).

The LAL assay has become the method of choice for detection and quantification of LPS in biological fluids (11-13). The validity of the LAL assay in measuring plasma concentrations of LPS is, however, still debated (14). Confirmatory chemical tests have not been applicable to plasma from patients with Gram-negative infections owing to the lack of sensitivity and cumbersome methodology (15-17). In human Gram-negative septicemia, the concentration of LPS in heparin plasma is usually below $100 \mathrm{ng} /$ liter (1 endotoxin unit [EU] per milliliter) as measured with the LAL assay $(18,19)$. In some cases of fulminant meningococcal septicemia much higher levels of LPS have been measured $(3,20)$. Plasma samples from such patients have made it possible for us to confirm chemically the presence of neisserial lipid $A$ in these cases by employing gas chromatography and mass spectrometry (GC-MS). We have, furthermore, demonstrated a close correlation between the two assay methods.

Little is known about the physicochemical state of LPS in human disease. Several research groups have documented a reproducible pattern of reactions involving disaggregation of LPS, and formation of LPS-lipoprotein complexes, notably with high density lipoprotein (HDL) in animal model studies (21-24). In these experiments purified LPS, while in a physicochemical state not likely to occur in vivo, have been employed. Several other plasma proteins have, furthermore, the ability to complex with LPS (24-29). In vitro studies of Escherichia coli and Salmonella abortus equi suggest that only 15-30\% of LPS present in the outer membrane can be released by the action of normal human serum $(30,31)$. Preincubation with various antibiotics does not significantly alter this percentage (30). Serumreleased LPS exhibit a reduced toxicity and enhanced immunomodulatory effects compared with phenol-water-extracted LPS (32). The physicochemical state and biological effects of circulating LPS, while still integrated in the outer membrane of the bacteria, have not been studied as extensively in animal models. By using a rat model more closely simulating the natural infection, Munford et al. (33) compared the intravascular behavior of purified LPS of Salmonella typhimurium to that of native LPS, i.e., LPS integrated in the outer cell membrane. Approximately $50 \%$ of purified LPS formed complexes with HDL, whereas LPS still integrated in the outer membranes or 
in whole bacteria exhibited $<10 \%$ HDL binding which strongly influenced the kinetics of elimination and tissue uptake of LPS (33). In that LPS complexed with lipoproteins reveal a reduced toxicity which parallels a reduced ability to elicit cytokine responses in cell model systems, lack of such binding may have important biological consequences in vivo (34-40).

In our study we have examined whether the LAL-reactive material in patients plasmas defined as bioreactive, native LPS primarily was present as complexes with lipoproteins or as LPS presumably still integrated in outer membrane structures, alternatively as large, aggregated LPS complexes. It has been known for some time that Neisseria meningitidis produces surplus outer membrane material (protrusions and vesicles also called "blebs") containing LPS during in vitro log-phase growth (4143). The presence of similar structures during growth in human blood has not yet been documented. To test these hypotheses we have centrifuged seven septic shock plasmas collected from lethal cases at $103,000 \mathrm{~g}$ for 15 and $60 \mathrm{~min}$, respectively. The sedimentation behavior of bioreactive LPS was related to that of HDL, LDL, and several other plasma proteins with known sedimentation coefficients. The results indicated that a major part of native LPS sedimented much faster than $\alpha_{2}$-macroglobulin (sedimentation coefficient of 19.6 Svedberg units [S] in serum). This sedimentation behavior was clearly different from that of HDL and LDL, and other potential carrier proteins of released LPS with low sedimentation coefficients. LPS activity related to outer membrane fragments and vesicles that were detected by electron microscopy or highly aggregated LPS released from the outer membrane may, thus, explain our centrifugation results.

\section{Methods}

Patient plasma. Blood samples were collected shortly after hospital admission from patients with bacteriologically verified fulminant meningococcal septicemia (3). Heparinized (15 U/ml) LPS-free vacuum tubes were used, and the blood was centrifuged at $1,400 \mathrm{~g}$ for $10 \mathrm{~min}$, thereby removing blood cells and $>99 \%$ of colony forming units (CFU) from the supernatant. The supernatant was pipetted off into cryotubes (Nunc, Roskilde, Denmark) and stored at $-70^{\circ} \mathrm{C}$ until analyzed.

LAL chromogenic substrate assay. The procedure has been described in detail elsewhere (3). The detection limit was $25 \mathrm{ng} / \mathrm{liter}$ with Escherichia coli 055B5 LPS (9 EU/ng; Whittaker Bioproducts, Walkersville, MD) as reference endotoxin. Duplicate samples of LPS-containing material were always retested in separate runs and the mean value was used. Similar recoveries of LPS in the LAL assay were obtained for vigorously vortexed or briefly ultrasound-treated (30 s, 35 kHz, 240 W; Bandelin Sonorex, Berlin, FRG) plasma samples (data not shown). Sonicated suspensions $\left(>10^{8} \mathrm{cfu} / \mathrm{ml}\right)$ of Streptococcus pyogenes (groups A, B, and D), S. pneumoniae, Staphylococcus aureus, and $S$. epidermidis were all negative in the $\mathrm{LAL}$ assay.

Purification of meningococcal LPS. N. meningitidis strain 44/76 (B:15:P1.16, "Norwegian clone" belonging to the ET 5 complex by isoenzyme genotyping) (44) was grown in a fermenter and LPS were isolated by three successive extractions: $0.1 \mathrm{M}$ Tris- $\mathrm{HCl}, \mathrm{pH} \mathrm{8.8,} \mathrm{con-}$ taining $0.5 \%$ deoxycholate and $10 \mathrm{mM}$ EDTA; hot phenol (45); phenol-chloroform-hexane (46). In the latter method hexane was substituted for petroleum ether. The extracted LPS were further purified by chromatography on Sephadex G-75 in the presence of $0.5 \%$ deoxycholate, whereafter LPS were precipitated from the eluate at $-20^{\circ} \mathrm{C}$ with $4 \mathrm{vol}$ of ethanol containing $0.05 \mathrm{M} \mathrm{NaCl}$. The final product contained $<0.3 \%$ protein and was without detectable nucleic acids (by ultraviolet and by $\mathrm{GC}$ for ribose). For the LAL assay, one vial of stock solution of LPS $\left(1 \mathrm{mg} / \mathrm{ml}\right.$ in $\left.\mathrm{H}_{2} \mathrm{O}\right)$, stored at $-20^{\circ} \mathrm{C}$, was thawed at room temperature, vigorously vortexed for $3 \mathrm{~min}$, and further diluted to $2 \mu \mathrm{g} / \mathrm{l}$ in LPS-free $\mathrm{H}_{2} \mathrm{O}$.

Determination of 3-hydroxy lauric acid (3-OH-12:0). Neisserial LPS contains 2 mol 3-OH-12:0, $O$-ester-linked to the glucosamine disaccharide backbone of the lipid A part $(47,48)$. This fatty acid is relatively specific for neisserial LPS, e.g., not present in LPS derived from Haemophilus influenzae and Enterobacteriaceae (49). It is easily cleaved off by mild acid treatment and is thus suitable as a specific marker for neisserial endotoxin. The other hydroxy fatty acid, 3-hydroxy myristic acid (3-OH-14:0), is linked in a more stable amide linkage, and is thus grossly unreleased at the conditions used.

Samples $(200 \mu \mathrm{l})$ of plasma from patients and of pooled plasma from healthy controls were transferred to $1-\mathrm{ml}$ glass vials with teflonlined screw caps and lyophilized for $1 \mathrm{~h}$. To this material was added 20 $\mu \mathrm{l}$ of 3-hydroxy decanoic acid (3-OH-10:0) methyl ester (Applied Science Corp., Philadelphia, PA; $0.5 \mu \mathrm{g} / \mathrm{ml}$ in methanol) as internal standard and $400 \mu \mathrm{l}$ of $2 \mathrm{M} \mathrm{HCl}$ in dry methanol. This mixture was heated at $85^{\circ} \mathrm{C}$ for $2 \mathrm{~h}$ for cleavage of $O$-ester linkages (50). The liberated fatty acid methyl esters were then extracted twice with hexane (2 vol) after addition of 1 vol of LPS-free water, half-saturated with $\mathrm{NaCl}$. This crude extract of lipophilic material was concentrated to $\sim 0.2 \mathrm{ml}$ and then applied to a silica extraction column (Bond Elut, Analytichem Int., Harbor City, CA) prewashed with 2 vol each of hexane, methylene chloride, and hexane/methylene chloride (1:1). The nonpolar constituents were first removed by $3 \mathrm{ml}$ of hexane- $\mathrm{CH}_{2} \mathrm{Cl}_{2}(1: 1)$, whereafter $3 \mathrm{ml}$ of dry diethyl ether eluted the polar constituents, including the 3-hydroxy fatty acid methyl esters. The solvent was removed by nitrogen before derivatization of the residue.

Free hydroxyl groups were derivatized using $50 \mu \mathrm{l}$ of the following trimethylsilyl reagent, bis-trimethylsilyl-trifluoroacetamide/acetonitrile/pyridine (50:40:10) (Supelco SA, Gland, Switzerland), and heating at $85 \mathrm{C}$ for $30 \mathrm{~min} .2 \mu \mathrm{l}$ of this reaction mixture was injected into the gas chromatograph-mass spectrometer instrument.

$G C-M S$. GC-MS analyses were carried out by using a gas chromatograph (model 3300, Varian, Walnut Creek, CA) with a (25 m $\times 0.2$ $\mathrm{mm}$ ) fused-silica SE-30 column (Hewlett-Packard Co., Palo Alto, CA) connected to a mass spectrometer (Ion-Trap 700, Finnigan-MAT, San Jose, CA). The instrument was used with electron-impact ionization at $70-\mathrm{eV}$ ion energy and in selective ion mode in the mass range 170-350. Calculations were based on the mass spectrometric fragmentation pattern of the trimethylsilyl-derivatized methyl esters of 3-OH-12:0 and the internal standard, 3-OH-10:0, respectively. These two fatty acid derivatives yielded related mass spectra characterized by an abundant $(\mathrm{M}-15)^{+}$ion, useful for quantification of the parent compounds (15, 16). A standard curve was constructed (by the Ion-trap software) on the basis of four solutions of 3-OH-12:0 in the concentrations of 100, 50, 25 , and $5 \mu \mathrm{g} /$ liter, respectively, each containing $50 \mu \mathrm{g} /$ liter of IS. Injection of $2 \mu \mathrm{l}$ of the $50-\mu \mathrm{l}$ samples provided signals corresponding to 200 , 100,50 , and $10 \mathrm{pg}$ of 3-OH-12:0, respectively, and to $100 \mathrm{pg}$ of internal standard.

The molecular mass of meningococcal LPS varies within certain limits (3.2-7.1 kD) owing to heterogeneity in size of the carbohydrate chain depending on strain, i.e., immunotype, and to microheterogeneity of the LPS related to growth conditions (51-53). For calculations in this study, we have chosen a molecular mass of $5.0 \mathrm{kD}$.

Ultracentrifugation of plasma containing native LPS. Heparinized plasma was collected from seven patients with lethal meningococcal endotoxemia (see Tables II and IV). The LPS levels ranged from 204 to $7.1 \mu \mathrm{g} /$ liter. All samples were vigorously vortexed for $3 \mathrm{~min}$. Subsequently, $150 \mu \mathrm{l}$ was transferred to LPS-free plastic tubes, and centrifuged in an Airfuge (Beckman Instruments, Inc., Palo Alto, CA) at $103,000 \mathrm{~g}$ for 15 or $60 \mathrm{~min}$ at $20^{\circ} \mathrm{C}$. The upper $50 \mu \mathrm{l}$ was pipetted off for quantification of plasma proteins and of LPS by LAL. The LPS contents were measured in the top, middle, and bottom $50-\mu$ l fractions. In three additional patient plasmas, the sedimentation behavior of native LPS and apolipoproteins (apo) A1 and B100 representing HDL and 
LDL, respectively, was determined simultaneously. Albumin $(69 \mathrm{kD}$, 4.6 S), fibrinogen (340 kD, 7.63 S), and $\alpha_{2}$-macroglobulin (725 kD, 19.6 S)) were used as markers to estimate the effect of ultracentrifugation on plasma components in donor plasma or serum with known sedimentation coefficients at $20^{\circ} \mathrm{C}(54)$.

Ultracentrifugation of purified meningococcal LPS added to acutephase plasma. Two concentrations of purified meningococcal LPS were added to each of three acute-phase plasma samples collected from three different patients with systemic meningococcal disease without detectable native circulating LPS in plasma. The final concentrations of LPS in the six samples ranged from 1.1 to $74 \mu \mathrm{g} /$ liter by LAL. The samples were ultrasound-treated (vide supra) at $37^{\circ} \mathrm{C}$ for $180 \mathrm{~s}$, incubated, and agitated at $37^{\circ} \mathrm{C}$ for $1 \mathrm{~h}$, and $150 \mu \mathrm{l}$ was transferred to an LPS-free plastic tube and centrifuged at $103,000 \mathrm{~g}\left(20^{\circ} \mathrm{C}\right)$ for $15 \mathrm{~min}$. The LPS concentrations were measured in the top, middle, and bottom $50-\mu l$ fractions.

Quantification of plasma proteins. Apo A1 and B100 were quantified immunoturbidometrically using a seven-point standard curve and an automated enzyme analyzer (Cobas Fara, Hoffmann LaRoche, Basel, Switzerland) essentially according to the manufacturer. Reagents for measurement of apo Al were purchased from Orion Diagnostica, Espoo, Finland, and for apo B100 from Behring Werke AG, Marburg, FRG. The interassay coefficient of variation $(\mathrm{CV})$ was $7.4 \%$ for apo $\mathrm{Al}$ and 3.4\% for apo B100. Albumin was measured with the bromcresol purple method (CV 3.6\%) (55). Fibrinogen was measured according to Clauss (CV 7.5\%) (56), and $\alpha_{2}$-macroglobulin with rocket immunoelectrophoresis.

Preparation of plasma for electron microscopy. Three heparinized patient plasmas containing 50,19 , and $8.7 \mu \mathrm{g} /$ liter, respectively, were studied. In addition, one sample containing the supernatant of $N$. meningitidis (B:15:P.1:16, isolate from patient 1 in Tables I and II) grown overnight in decomplemented $\left(56^{\circ} \mathrm{C}, 30 \mathrm{~min}\right)$, undiluted human heparinized plasma and centrifuged at $1,400 \mathrm{~g}$ for $10 \mathrm{~min}$, was investigated. Ultracentrifugation was performed as above in LPS-free plastic tubes. $250 \mu \mathrm{l}$ was pipetted into the tubes and centrifuged (first run) at 103,000 $g$ for $60 \mathrm{~min}$, and four fractions (called fractions 1, 2, 3, and 4) from the top to the bottom were transferred into separate, LPS-free glass tubes, diluted $1+9$ with LPS-free $\mathrm{H}_{2} \mathrm{O}$ and vortexed for $3 \mathrm{~min} .250 \mu \mathrm{l}$ of the diluted fraction 4 was centrifuged (second run) at $103,000 \mathrm{~g}$ for $60 \mathrm{~min}$ and four fractions from the top to bottom (called fractions 4.1, 4.2, 4.3, and 4.4) were pipetted into LPS-free glass tubes, diluted $1+4$ with $\mathrm{H}_{2} \mathrm{O}$, and vortexed for $3 \mathrm{~min}$. The diluted fraction 4.4 was centrifuged (third run) at $103,000 \mathrm{~g}$ for $60 \mathrm{~min}$ and divided into four fractions called 4.4.1, 4.4.2, 4.4.3, and 4.4.4 as above. The fraction 4.4.4 (final plasma concentration 1:50) was used for electron microscopy.

Electron microscopy. A drop of the four solutions containing fraction 4.4.4 was applied to carbon-filmed, glow-discharged grids, left for $1 \mathrm{~min}$, and stained with $0.5 \%$ phosphotungstic acid (NaPT), $\mathrm{pH} 7.0$, for another min. After blotting off and air drying, the specimens were examined in an electron microscope (model 100B, Japan Electron Optical Laboratory, Tokyo, Japan) at $80 \mathrm{KeV}$, operated with the liquid nitrogen anticontamination trap.

Statistical methods. Statistical differences between the groups were assessed with Mann-Whitney's U test. The correlation coefficient was calculated according to Pearson with corresponding $t$ test. $P<.05$ was considered statistically significant.

\section{Results}

Comparison of LPS quantified with LAL assay and GC-MS. Quantifiable amounts of 3-OH-12:0 were detected in all five plasma samples with high LPS levels ( $>5 \mu \mathrm{g} /$ liter by LAL) that were selected for GC-MS analysis (Table I, Fig. 1). The results obtained with the two methods were of the same magnitude and revealed a high correlation coefficient $(r=0.98, P=0.006)$ (Table I). The lowest values found by the GC-MS method may, however, be of limited accuracy, owing to a background level
Table I. Plasma Levels of LPS in Four Lethal Cases of Systemic Meningococcal Disease Determined by Chromogenic LAL Assay and GC-MS

\begin{tabular}{lrr}
\hline & \multicolumn{2}{c}{ Concentration of LPS } \\
\cline { 2 - 3 } \multicolumn{1}{c}{ Patient } & LAL & GC-MS \\
\hline & & $\mu g / l i t e r$ \\
1 (on admission) & 170 & 140 \\
1 (4 h and 20 min later) & 50 & 24 \\
2 & 19 & 13 \\
3 & 6 & 15 \\
4 & 11 & 14
\end{tabular}

$r=0.98, P=0.006$

of 3-hydroxy fatty acids of unknown origin in plasma samples, as observed when negative plasmas were taken through the complete procedure (data not shown). To date we regard 10 $\mu \mathrm{g} /$ liter as a practical detection limit for LPS with GC-MS, using a final sample volume of 50 - and $2-\mu$ injections. In addition to 3-OH-12:0, the more usual LPS-specific fatty acid 3$\mathrm{OH}-14: 0$ was detected in all patient samples (data not shown). Notable is the high level of LPS in patient 1 , and the significant fall $4 \mathrm{~h}$ and $20 \mathrm{~min}$ after hospital admission, measured by both methods (Table I).

Comparison of LAL activity of $E$. coli and $N$. meningitidis LPS. Standard curves ranging from 2.5 to $40 \mathrm{ng} /$ liter were prepared from stock solutions $\left(2 \mu \mathrm{g} /\right.$ liter $\left.\mathrm{H}_{2} \mathrm{O}\right)$ of either $E$. coli 055B5 (9 EU/ng) or $N$. meningitidis LPS which is the Norwegian reference LPS prepared from strain 44/76 (B:15:P1.16, ET-5 complex) (vide above). The results based on three separate runs were $2.5(2.6 \pm 1.2), 5(6.9 \pm 1.8), 10(12 \pm 2.5), 20$ $(24 \pm 6.7)$, and $40(48 \pm 9.8) \mu \mathrm{g} /$ liter ( $N$. meningitidis LPS values in parenthesis, mean $\pm \mathrm{SE}$ ), suggesting similar biological activity in the LAL assay of the two preparations.

Effect of ultracentrifugation on native LPS in shock plasma. The LPS levels before centrifugation were 204, 31.9, 22.3, 15.5,

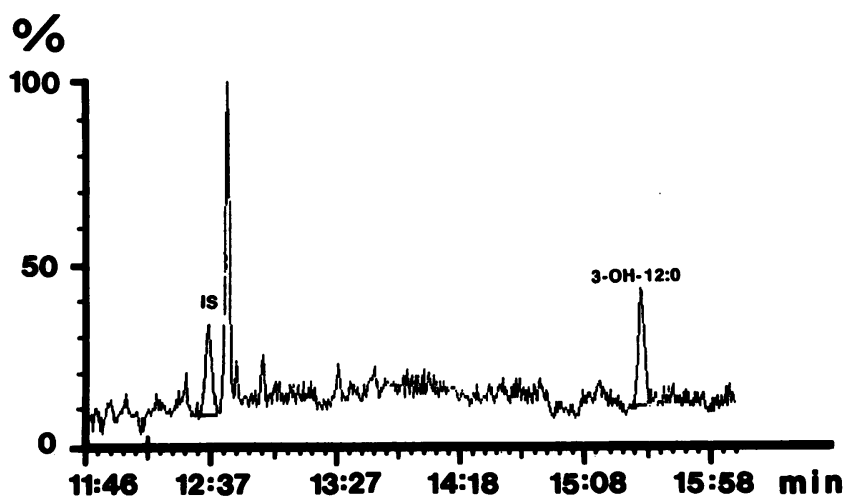

Figure 1. GC-MS-selected ion profile of plasma from a patient with fulminant meningococcal septicemia. Ester-linked fatty acids were released by brief acidic methanolysis and extracted/separated on a silica column, and the hydroxy-fatty acid fraction was trimethylsilyl-derivatized. The ion $m / z 287$ (M-15) was selected for analysis of 3-OH-12:0. The internal standard 3-OH-10:0 was added before methanolysis, and monitored by $m / z 259$ (M-15) during the same run. The major peak eluting just after the internal standard is of unknown origin. 
Table II. Effect of Ultracentrifugation $\left(103,000 \mathrm{~g}, 20^{\circ} \mathrm{C}\right)$ on Native LPS in Heparin Plasma (A) Collected from Seven Patients with Lethal Meningococcal Endotoxemia Centrifuged for $15 \mathrm{~min}$, or (B) Collected from Six Patients with Lethal Meningococcal Endotoxemia Centrifuged for $60 \mathrm{~min}$

\begin{tabular}{|c|c|c|c|c|c|c|c|c|c|}
\hline A. Patient & 1 & 2 & 3 & & 4 & 5 & 6 & 7 & Mean \pm SE \\
\hline Initial level $(\mu g /$ liter $)$ & 204 & 31.9 & 22.3 & & 15.5 & 9.8 & 8.1 & 7.1 & \\
\hline Total recovery (\%) & 40 & 96 & 77 & & 107 & 57 & 124 & 87 & $84 \pm 11$ \\
\hline \multicolumn{10}{|c|}{$\begin{array}{l}\text { Distribution after centrigation } \\
\text { for } 15 \mathrm{~min}\end{array}$} \\
\hline Top (\%) & 4 & 5 & 7 & & 6 & 7 & 14 & 13 & $8 \pm 1$ \\
\hline Middle (\%) & 8 & 13 & 12 & & 8 & 12 & 23 & 15 & $13 \pm 2$ \\
\hline Bottom (\%) & 88 & 82 & 82 & & 86 & 82 & 63 & 72 & $79 \pm 3$ \\
\hline B. Patient & 1 & 2 & & 3 & & 4 & 5 & 6 & Mean \pm SE \\
\hline Initial level $(\mu g /$ liter $)$ & 204 & 38.9 & & 31.9 & & 23.8 & 22.3 & 9.8 & \\
\hline Total recovery (\%) & 58 & 50 & & 52 & & 43 & 69 & 52 & $54 \pm 4$ \\
\hline \multicolumn{10}{|c|}{$\begin{array}{l}\text { Distribution after centrifugation } \\
\text { for } 60 \mathrm{~min}\end{array}$} \\
\hline Top (\%) & 2 & 3 & & 0 & & 1 & 6 & 2 & $2 \pm 1$ \\
\hline Middle (\%) & 3 & 8 & & 10 & & 6 & 11 & 5 & $7 \pm 1$ \\
\hline Bottom (\%) & 95 & 89 & & 90 & & 93 & 83 & 93 & $91 \pm 2$ \\
\hline
\end{tabular}

Total recovery, given as mean $\pm \mathrm{SE}$, denotes the sum of the detected levels in top, middle, and bottom $50-\mu$ l fractions as compared with the initial level. The distribution in percent after centrifugation is indicated for the top, middle, and bottom $50-\mu$ l fractions.

9.8, 8.1, and $7.1 \mu \mathrm{g} /$ liter, respectively, for patients $1-7$. (Table II). After $15 \mathrm{~min}$ of centrifugation at $103,000 \mathrm{~g}$, the levels were measured in the top, middle, and bottom $50-\mu$ l fractions. The sum of these levels was defined as total recovery and expressed as the percentage of the level before centrifugation. The total recovery ranged from $40 \%$ to $124 \%$ (Table II). For the group of seven specimens the mean \pm SE total recovery was $84 \pm 11 \%$. The distribution of the total recovered LPS in the top, middle, and bottom $50-\mu l$ fractions was calculated by defining the total recovered level as $100 \%$ (Table II). The top 50- $\mu$ l fraction contained $8 \pm 1 \%$ (mean \pm SE, $n=7)$ of the recovered LPS whereas $79 \pm 3 \%($ mean \pm SE, $n=7)$ were recovered in the bottom $50-\mu 1$ fraction after $15 \mathrm{~min}$ of centrifugation (Table II). After $60 \mathrm{~min}$ of centrifugation, $2 \pm 1 \%$ (mean \pm SE, $n=6$ ) and $91 \pm 2 \%$ of the recovered native LPS were found in the top and bottom fractions, respectively (Table II). The total recovery of native LPS after centrifugation decreased with increasing levels of LPS in the samples before centrifugation. This may relate to extensive dilution (1:1,000 or more), adherence of LPS to the tube wall, and compression of the LPS complexes during centrifugation at $103,000 \mathrm{~g}$ making them less accessible for the LAL reagents.

Effect of ultracentrifugation on purified meningococcal LPS dissolved in acute phase plasma. The mean $\pm \mathrm{SE}$ total recovery of purified LPS dissolved in acute-phase plasma after $15 \mathrm{~min}$ of centrifugation was $87 \pm 13 \%$ (range $57-140 \%$ ). Of the recovered LPS, $10 \pm 3 \%($ mean \pm SE, $n=6)$ remained in the top $50 \mu$, whereas $78 \pm 6 \%$ were found in the bottom $50-\mu$ l fraction. The sedimentation patterns of six samples containing purified LPS added to acute-phase plasmas were not significantly different from the patterns observed for seven samples with native meningococcal LPS (Table II) $(P>0.1)$.

Effect of ultracentrifugation on reference proteins vs. native LPS in normal and shock plasma. The sedimentation behavior of albumin (4.6S), fibrinogen (7.6S), $\alpha_{2}$-macroglobulin (19.6S), apo $A 1$, and apo $B 100$ in normal donor plasma after centrifuga- tion at $103,000 \mathrm{~g}$ for 15 and $60 \mathrm{~min}$ was studied in samples collected from three different persons (Table III). The concentrations of HDL and LDL revealed only minor changes under these sedimentation conditions, whereas the concentrations of albumin, fibrinogen, and $\alpha_{2}$-macroglobulin decreased in the top fractions and increased in the bottom fractions corresponding to the sedimentation coefficients (Table III). The total recovery of the various proteins after centrifugation ranged from $89 \%$ to $109 \%$ (Table III). The sedimentation patterns of native LPS vs. HDL and LDL in three patient plasmas are given in Table IV.

Electron microscopy of ultracentrifuged patient plasma and cultivated $N$. meningitidis. Overlapping membrane fragments demonstrating a typical moiré pattern (57) were identified in plasma samples from three patients examined (Fig. $2 a$ ). Similar bacterial fragments were also detected in decomplemented human heparin plasma inoculated with meningococci and grown over night (data not shown). In one patient sample, a bacterium with multiple long membrane protrusions was observed (Fig. $2 b$ ) as has previously been demonstrated for meningococci grown under in vitro conditions (41-43). These observations suggest that formation of surplus outer membrane material by $N$. meningitidis multiplying in human blood really occurs in vivo.

\section{Discussion}

The results of this study suggest four conclusions. First, circulating endotoxin collected from patients developing fulminant meningococcal septicemia is derived from $N$. meningitidis and is not of enteric origin. Second, quantitative chemical detection of native LPS in patient plasmas was in accordance with measurement made with the LAL assay. Third, an extensive part of native meningococcal LPS appears to circulate as complexes with high sedimentation coefficients and outer membrane fragments were detected by electron microscopy in all 
Table III. Effect of Ultracentrifugation $\left(103,000 \mathrm{~g}, 20^{\circ} \mathrm{C}\right)$ on Five Plasma Proteins

in Normal Human Plasma Centrifuged for 15 and $60 \mathrm{~min}$

\begin{tabular}{|c|c|c|c|c|c|}
\hline & $\begin{array}{l}\text { Albumin } \\
(4.6 \mathrm{~S})\end{array}$ & $\begin{array}{l}\text { Fibrinogen } \\
\quad(7.6 \mathrm{~S})\end{array}$ & $\begin{array}{c}\alpha_{2} \text {-macroglobin } \\
(19.6 \mathrm{~S})\end{array}$ & Apo Al & Apo B100 \\
\hline \multicolumn{6}{|c|}{ Plasma centrifuged for $15 \mathrm{~min}$} \\
\hline Total recovery (\%) & $98 \pm 1$ & $109 \pm 5$ & $106 \pm 23$ & $100 \pm 2$ & $102 \pm 3$ \\
\hline \multicolumn{6}{|l|}{ Fraction } \\
\hline Top (\%) & $29 \pm 0$ & $24 \pm 2$ & $19 \pm 1$ & $32 \pm 1$ & $36 \pm 1$ \\
\hline Middle (\%) & $34 \pm 0$ & $35 \pm 2$ & $32 \pm 2$ & $34 \pm 0$ & $33 \pm 0$ \\
\hline Bottom (\%) & $37 \pm 0$ & $41 \pm 0$ & $49 \pm 2$ & $34 \pm 1$ & $31 \pm 1$ \\
\hline \multicolumn{6}{|c|}{ Plasma centrifuged for $60 \mathrm{~min}$} \\
\hline Total recovery (\%) & $99 \pm 2$ & $89 \pm 1$ & $97 \pm 33$ & $99 \pm 1$ & $98 \pm 2$ \\
\hline \multicolumn{6}{|l|}{ Fraction } \\
\hline Top (\%) & $20 \pm 0$ & $9 \pm 1$ & $0 \pm 0$ & $27 \pm 0$ & $40 \pm 1$ \\
\hline Middle (\%) & $34 \pm 1$ & $31 \pm 1$ & $18 \pm 1$ & $35 \pm 0$ & $34 \pm 1$ \\
\hline Bottom (\%) & $46 \pm 1$ & $59 \pm 1$ & $82 \pm 1$ & $38 \pm 0$ & $26 \pm 1$ \\
\hline
\end{tabular}

Total recovery and the distribution in top, middle, and bottom 50- $\mu$ l fractions as defined in Table II. S denotes Svedberg units in plasma or serum.

specimens examined. Fourth, the sedimentation behavior of native LPS during ultracentrifugation was clearly different from the sedimentation behavior of HDL and LDL, suggesting that most of the bioreactive LPS were not primarily complexed with these lipoproteins.

The results from the gas chromatographic and mass spectrometric study suggest that the LAL activity in patient plasmas is related to lipid A derived from neisserial LPS and not from translocated bacteria of enteric origin that has escaped into the general circulation during the septic shock state. This conclusion is based on the identification of the 3-OH-12:0, which, although also found in certain Pseudomonas species is not present in LPS of enterobacteria and is relatively specific for neisserial LPS (49). This conclusion agrees with two previous observations made in fatal septic shock patients. Fatal septicemia caused by Streptococcus pneumoniae in splenectomized patients reveals a clinical picture similar in many ways to fulminant meningococcal septicemia with irreversible septic shock, extensive coagulopathy, and multiple organ failure. In our laboratory LAL assays of sequentially collected plasma samples from such cases have always been negative ( $<25 \mathrm{ng} /$ liter) (3), suggesting that severe septic shock per se does not necessarily facilitate gut-derived systemic endotoxemia in humans. Thus, the multiple organ failure syndrome related to $S$. pneumoniae is presumably caused by Gram-positive cell components. Furthermore, the constant clearance rate of LPS resulting in complete elimination, which is observed in patients with fulminant meningococcal septicemia (3), would be difficult to explain if LPS was gut-derived. $N$. meningitidis was the only bacterium isolated in blood cultures from the four patients studied (3).

Chemical identification of LPS in plasma is not feasible in most cases of human Gram-negative sepsis owing to levels far below the detection limit of GC-MS methods $(15,16,18,19)$. Fulminant meningococcal septicemia is a unique disease inasmuch as the high levels of LPS in plasma that make chemical detection and identification of neisserial lipid A possible are concerned. Importantly, there was a close correlation between the plasma levels of LPS, as determined with the LAL assay measuring lipid A activity and the quantitative chemical detection of the lipid A-derived 3-OH-12:0 (Table I). The declining
LPS levels consistently observed in these patients by using the LAL assay (3) were supported by GC-MS analysis of one patient plasma with an exceptionally high initial LPS content (Table I). This observation, if confirmed in future studies, suggests that most of the LPS-containing material is removed from the circulation during fulminant meningococcal septicemia and that lipid A is not merely concealed, i.e., "detoxified," by formation of complexes with plasma proteins.

The 3-OH-14:0, which also derives from meningococcal lipid A, was detected in all five patient plasmas examined (data not shown). This fatty acid is amide-linked in neisserial LPS and is not released to a high degree by the method employed. The 3-OH-14:0 is a constituent of many bacteria and could therefore not be used as a specific neisserial lipid A marker. It is

Table IV. Effect of Ultracentrifugation $\left(103,000 \mathrm{~g}\right.$ at $\left.20^{\circ} \mathrm{C}\right)$ on Apo A1, Apo B100, and Native LPS in Plasma from Three Patients with Lethal Endotoxemia Centrifuged for 15 and $60 \mathrm{~min}$

\begin{tabular}{lccc}
\hline & $\begin{array}{c}\text { Apo A1 } \\
(n=3) \\
\text { Mean } \pm \text { SE }\end{array}$ & $\begin{array}{c}\text { Apo B100 } \\
(n=3) \\
\text { Mean } \pm \text { SE }\end{array}$ & $\begin{array}{c}\text { LPS } \\
(n=3) \\
\text { Mean } \pm \text { SE }\end{array}$ \\
\hline $\begin{array}{l}\text { Plasma centrifuged } \\
\text { for 15 min }\end{array}$ & & & \\
$\quad \begin{array}{l}\text { Total recovery (\%) } \\
\text { Fraction }\end{array}$ & $105 \pm 5$ & $99 \pm 2$ & $105 \pm 11$ \\
$\quad \begin{array}{l}\text { Top (\%) } \\
\text { Middle (\%) }\end{array}$ & $34 \pm 2$ & $35 \pm 1$ & $11 \pm 3$ \\
$\quad$ Bottom (\%) & $32 \pm 1$ & $33 \pm 1$ & $15 \pm 4$ \\
$\begin{array}{l}\text { Plasma centrifuged } \\
\text { for 60 min }\end{array}$ & $34 \pm 0$ & $32 \pm 1$ & $74 \pm 7$ \\
$\quad$ Total recovery (\%) & & & \\
Fraction & $99 \pm 1$ & $104 \pm 0$ & $48 \pm 3$ \\
Top (\%) & & & \\
Middle (\%) & $30 \pm 0$ & $32 \pm 2$ & $1 \pm 1$ \\
Bottom (\%) & $33 \pm 0$ & $35 \pm 1$ & $8 \pm 1$ \\
& $36 \pm 0$ & $33 \pm 2$ & $91 \pm 1$ \\
\hline
\end{tabular}

Total recovery and the distribution in top, middle, and bottom $50-\mu 1$ fractions as defined in Table II. 

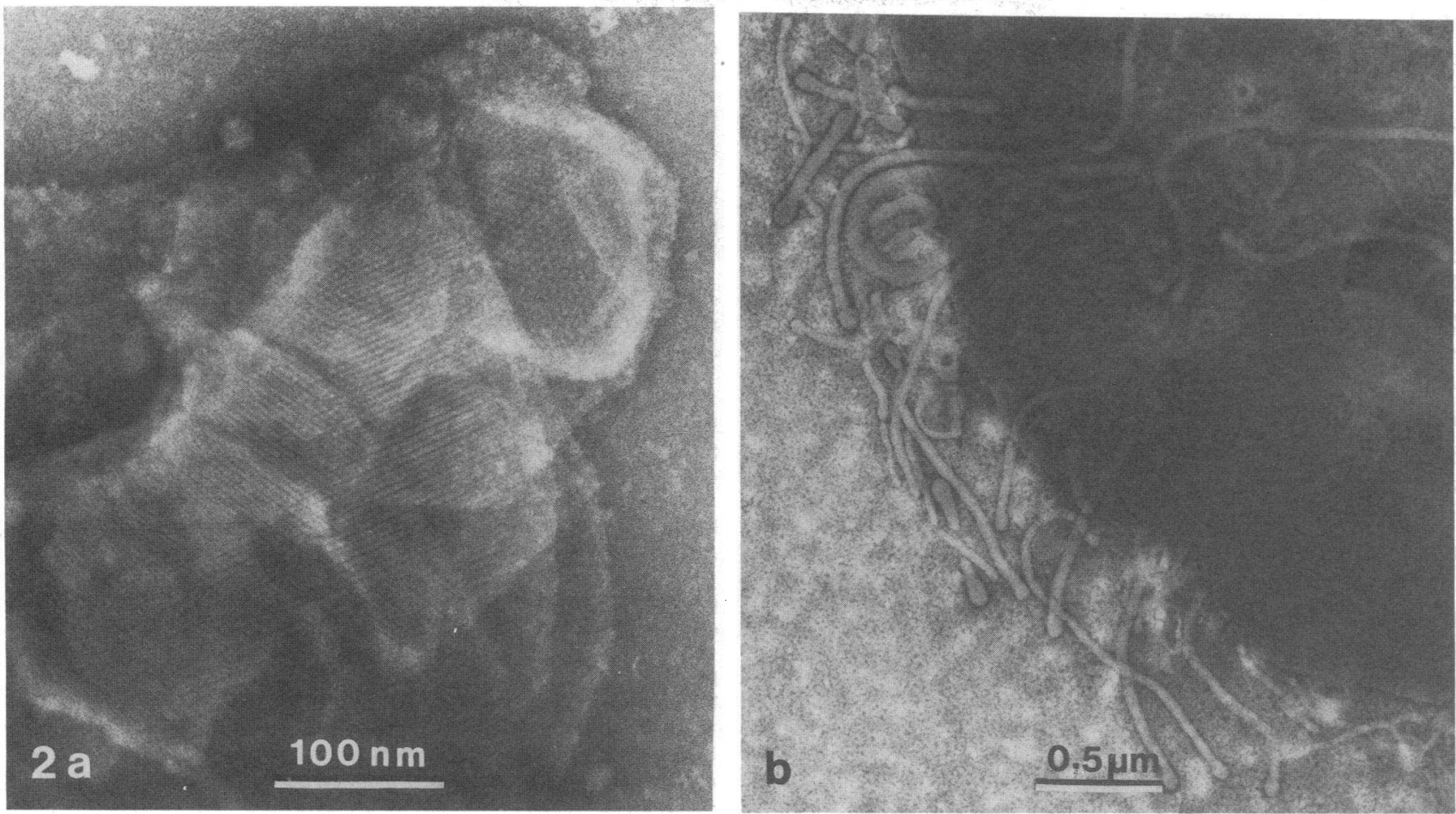

Figure 2. Transmission electron micrographs of negatively stained $(0.5 \% \mathrm{NaPT}, \mathrm{pH} 7.0)$ bacterial membranes found after ultracentrifugation at $103,000 \mathrm{~g}$ for $60 \mathrm{~min}$ (three runs). (a) Microbial unit membrane showing the typical moiré pattern from overlapping membrane fragments. The fragments were detected after ultracentrifugation in all lethal meningococcal shock plasmas $(n=3)$ examined. $\times 200,000$. $(b)$ Part of a microbe with multiple long membrane protrusions, found in one patient plasma. $\times 30,000$.

also detected in normal control plasma, although at a much lower level than observed in plasma from patients with fulminant meningococcal septicemia. The origin of 3-OH-14:0 in normal donor plasma is unknown.

To our knowledge, this is the first observation confirming quantitatively the results obtained with the LAL assay in patient plasmas with a chemical method. For clinical use the LAL assay remains the method of choice for detection of LPS owing to its superior sensitivity and applicability. The close quantitative measurements between the LAL assay and GC-MS suggests that most of the LPS present in circulation of these patients is biologically active, at least in the LAL assay. However, because of the low concentrations of 3-OH-12:0 in our patients, further chemical studies are needed before firm conclusions about the quantitative aspect of this measurement can be made.

The centrifugation studies suggest that native LPS circulate as complexes with high sedimentation coefficients. When plasmas from seven different patients were ultracentrifuged at $103,000 \mathrm{~g}$ for $15 \mathrm{~min}$, only $8 \pm 11 \%$ of the recovered native LPS was detected in the upper one-third of the centrifuged volume, whereas $79 \pm 3 \%$ were recovered in the lower one-third volume. (Tables II and IV). A particle or molecule, which transverses the distance from the minimum to the maximum radius within $15 \mathrm{~min}$ at $103,000 \mathrm{~g}$, is expected to have a sedimentation coefficient $\geq 68 \mathrm{~S}$ (58). LAL activity related to outer membrane vesicles and disintegrated bacteria could explain our findings by centrifugation (42). In line with this is our observation that electron microscopic examination of ultracentrifuged septic shock plasma identified multiple outer membrane fragments (Fig. $2 a$ ). Identical fragments were found in ultracentrifuged supernatant of meningococci grown in decomplemented, heparinized human plasma. Furthermore, one of three patients plasmas examined contained a bacterium covered with multiple, long membrane protrusions (Fig. $2 b$ ), indicating that production of surplus outer membrane really occurs in vivo. In vitro experiments have shown that meningococcal LPS still integrated in the bacterial outer membrane reveal considerable biological activity (17).

However, because purified meningococcal LPS prepared from the disease-causing clone and dissolved in acute-phase plasma revealed a sedimentation pattern similar to the one observed for native LPS, the formation of LPS aggregates in patient plasma could be an alternative explanation of our centrifugation data. Rough neisserial LPS dissolved in an aqueous plasma solutions tend to form large aggregates owing to the hydrophobic lipid A. The ability of human serum to release LPS from meningococci has not yet been studied in detail, and results obtained for $E$. coli and $S$. abortus equi may not necessarily apply to $N$. meningitidis. Thus, whether or not LPS released from the outer membrane and aggregated in patients plasma, can partly explain our results awaits further investigation.

We had expected to find high levels of LPS in the top fractions after centrifugation if most of the bioreactive native LPS had been present as detached LPS molecules associated with lipoproteins. The centrifugation conditions employed in this study did not significantly change the concentrations of apo A1 and apo B100 after $15 \mathrm{~min}$, whereas only $8 \pm 1 \%$ of the bioreactive native LPS was recovered in the top fraction. The real amount of LPS in this fraction might, however, be significantly higher in that the LAL assay measures only biologically active 
LPS. Lipoproteins, LPS-binding protein, and possibly other proteins appear to "detoxify," i.e., reduce the biological reactivity of LPS by complex formation (21-23, 32, 34-36, 38-40). The close agreement between GC-MS and LAL methods in quantification of native LPS in these plasmas, however, suggests that most of the circulating LPS is biologically active.

To what extent other plasma proteins, i.e., immunoglobulins and complement components with high affinity for LPS (29), may be attached to bacterial fragments and LPS complexes has not been addressed in this study. More research is clearly needed before the physiochemical state of native LPS and the relationship to plasma proteins in shock plasma are fully understood. Our data suggest the animal models employing live bacteria may be more appropriate than purified LPS in discerning the in vivo state of native endotoxin in humans.

Fulminant meningococcal septicemia is the prototype of an acute, overwhelming Gram-negative septicemia. Detailed studies of these patients have given us a unique opportunity to elucidate various disease mechanisms related to LPS in humans. We hope that this knowledge will increase our ability to avoid or neutralize fatal LPS effects in the future.

\section{Acknowledgment}

We thank G. Becher, National Institute of Public Health, Oslo, for help with the mass spectrometric determinations.

\section{References}

1. Morrison, D. C., and R. J. Ulevitch. 1978. The effects of bacterial endotoxins on host mediation systems. Am. J. Pathol. 93:527-617.

2. Morrison, D. C., and J. L. Ryan. 1987. Endotoxins and disease mechanisms. Annu. Rev. Med. 38:417-432.

3. Brandtzaeg, P., P. Kierulf, P. Gaustad, A. Skulberg, J. N. Bruun, S. Halvorsen, and E. Sørensen E. 1989. Plasma endotoxin as a predictor of multiple organ failure and death in systemic meningococcal disease. J. Infect. Dis. 159:195-204.

4. Waage, A., P. Brandtzaeg, A. Halstensen, P. Kierulf, and T. Espevik. 1989. The complex pattern of cytokines in serum from patients with meningococcal septic shock. J. Exp. Med. 169:333-338.

5. Brandtzaeg, P., T. E. Mollnes, and P. Kierulf. 1989. Complement activation and endotoxin levels in systemic meningococcal disease. J. Infect. Dis. 160:58-65.

6. Brandtzaeg, P., P. M. Sandset, G. B. Joø, R. Øvstebø, U. Abildgaard, and P. Kierulf. 1989. The quantitative association of plasma endotoxin, antithrombin, protein $\mathrm{C}$, extrinsic pathway inhibitor and fibrinopeptide $\mathrm{A}$ in systemic meningococcal disease. Thromb. Res. 55:459-470.

7. Brandtzaeg, P., R. Øvstebø, B. Brusletto, and P. Kierulf. 1990. Plasminogen activator inhibitor 1 and 2, alpha-2-antiplasmin, plasminogen and endotoxin levels in systemic meningococcal disease. Thromb. Res. 57:271-278.

8. Brandtzaeg, P., O. Øktedalen, P. Kierulf, and P. K. Opstad. 1989. Elevated VIP and endotoxin plasma levels in human gram-negative septic shock. Regul. Pept. 24:37-44.

9. Brandtzaeg, P., A. Waage, T. E. Mollnes, O. Øktedalen, and P. Kierulf. 1991. Severe human septic shock involves more than tumor necrosis factor. In Bacterial Endotoxins: Cytokine Mediators and New Therapies for Sepsis. A. Sturk, S. J. H. van Deventer, J. W. ten Cate, H. R. Büller, L. G. Thijs, and J. Levin, editors. John Wiley \& Sons, Inc., New York. 25-42.

10. Brandtzaeg, P., and P. Kierulf. 1992. Endotoxin and meningococcemia: intravascular inflammation induced by native endotoxin in man. In Bacterial Endotoxic Lipopolysaccharides, Volume 2: Immunopharmacology and Pathophysiology. J. L. Ryan, and D. C. Morrison, editors. CRC Press, Boca Raton, FL. In press.

11. Levin, J., T. E. Poore, N. P. Zauber, and R. S. Oser. 1970. Detection of endotoxin in the blood of patients with sepsis due to Gram negative bacteria. $N$. Engl. J. Med. 283:1313-1316.

12. Iwanaga, S., T. Morita, and T. Harada. 1978. Chromogenic substrates for horseshoe crab clotting enzyme, its application for the assay of bacterial endotoxin. Haemostasis. 7:183-186.

13. Sturk, A., and J. W. ten Cate. 1985. Endotoxin testing revisited. Eur. J. Clin. Microbiol. 4:382-385.
14. Elin, R. J., and J. Hosseini. 1985. Clinical utility of the Limulus amoebocyte (LAL) test. In Bacterial Endotoxins. J. W. ten Cate, H. R. Büller, A. Sturk, J. Levin, editors. Alan R. Liss, Inc., New York. 307-324.

15. Maitra, S. K., M. C. Schotz, T. T. Yoshikawa, and L. B. Guze. 1978. Determination of lipid A and endotoxin in serum by mass spectroscopy. Proc. Natl. Acad. Sci. USA 75:3993-3997.

16. Maitra, S. A., R. Nachum, and F. C. Pearson. 1986. Establishment of beta-hydroxy fatty acids as chemical marker molecules for bacterial endotoxin by gas chromatography-mass spectrometry. Appl. Environm. Microbiol. 52:510514.

17. Bryn, K., O. Solberg, and B. M. Andersen. 1989. Endotoxin liberation studied by biological and chemical methods: chemical characterization of six meningococcal lipopolysaccharides. APMIS (Acta Pathol. Microbiol. Immunol. Scand.) 97:429-435.

18. McCartney, A. C., B. I. Piotrowicz, S. E. Edlin, and I. McA. Ledingham. 1987. Evaluation of the chromogenic Limulus lysate assay in septic shock. In Detection of Bacterial Endotoxins with the Limulus Amebocyte Lysate Test S. W. Watson, J. Levin, and T. J. Novitsky, editors. Alan R. Liss, Inc., New York. 459-474.

19. Van Deventer, S. J. H., H. R. Büller, J. W. ten Cate, A. Sturk, and W. Pauw. 1988. Endotoxaemia: an early predictor of septicaemia in febrile patients. Lancet. 1:605-609.

20. Harthug, S., B. Bjorvatn, and B. Østerud. 1983. Quantitation of endotoxin in blood from patients with meningococcal disease using a limulus lysate test in combination with chromogenic substrate. Infection. 11:192-195.

21. Ulevitch, R. J., A. R. Johnston, and D. B. Weinstein. 1979. New function for high density lipoproteins: their participation in intravascular reactions of bacterial lipopolysaccharides. J. Clin. Invest. 64:1516-1524.

22. Freudenberg, M. A., T. C. Bog-Hansen, U. Back, and C. Galanos. 1980 Interaction of lipopolysaccharides with plasma high-density lipoprotein in rats. Infect. Immun. 28:373-380.

23. Munford, R. S., J. M. Andersen, and J. M. Dietschy. 1981. Sites of tissue binding and uptake in vivo of bacterial lipopolysaccharide-high density lipoprotein complexes: studies in the rat and squirrel monkey. J. Clin. Invest. 68:15031513.

24. Galanos, C., E. T. Rietschel, O. Luderitz, and O. Westphal. 1971. Interaction of lipopolysaccharides and lipid A with complement. Eur. J. Biochem. 19:143-152.

25. Tobias, P. S., and R. J. Ulevitch. 1983. Control of lipopolysaccharide-high density lipoprotein binding by acute phase protein(s). J. Immunol. 131:19131916.

26. Tesh, V. L., S. W. Vukajlovich, and D. C. Morrison. 1988. Endotoxin interactions with serum proteins, relationship to biological activity. In Bacterial Endotoxins: Pathophysiological Effects, Clinical Significance, and Pharmacological Control. J. Levin, H. R. Büller, J. W. ten Cate, S. J. H. van Deventer, and A Sturk, editors. Alan R. Liss, Inc., New York. 47-62.

27. Berger, D., and H. G. Beger. 1988. Quantification of the endotoxin-binding capacity of human transferrin. In Bacterial Endotoxins: Pathophysiological Effects, Clinical Significance, and Pharmacological Control. J. Levin, H. R. Büller, J. W. ten Cate, S. J. H. van Deventer, and A. Sturk, editors. Alan R. Liss, Inc., New York. 115-124.

28. Tobias, P. S., J. C. Mathison, and R. Ulevitch. 1988. A family of lipopolysaccharide binding proteins involved in responses to gram-negative sepsis. J. Biol. Chem. 263:13479-13481

29. Morrison, D. C. 1990. Diversity of mammalian macromolecules which bind to bacterial lipopolysaccharides. In Endotoxin Research Series, Volume 1. A. Nowotny, J. J. Spitser, and E. J. Ziegler, editors. Elsevier Science Publishers B. V., Amsterdam. 183-189.

30. Tesh, V. L., and D. C. Morrison. 1988. The interaction of Escherichia coli with normal human serum: factors affecting the capacity of serum to mediate lipopolysaccharide release. Microb. Pathog. 4:175-187.

31. Freudenberg, M. A., U. Meier-Dieter, T. Staehelin, and C. Galanos. 1991. Analysis of LPS released from Salmonella abortus equi in human serum. Microb. Pathog. 10:93-104.

32. Tesh, V. L., and D. C. Morrison. 1988. The physical-chemical characterization and biological activity of serum released lipopolysaccharides. J. Immunol. 141:3523-3531.

33. Munford, R. S., C. L. Hall, J. M. Lipton, and J. M. Dietschy. 1982. Biological activity, lipoprotein-binding behavior, and in vivo disposition of extracted and native forms of Salmonella typhimurium lipopolysaccharides. J. Clin. Invest. 70:877-888.

34. Skarnes, R. C., F. S. Rosen, M. J. Shear, and M. Landy. 1958. Inactivation of endotoxin by a humoral component. II. Interaction of endotoxin with serum and plasma. J. Exp. Med. 108:685-700.

35. Skarnes, R. C. 1985. In vivo distribution and detoxification of endotoxins In Cellular Biology of Endotoxin. L. J. Berry, editor. Elsevier Science Publishers, B.V., Amsterdam. 56-81.

36. Warren, H. S., C. V. Knights, and G. R. Siber. 1986. Neutralization and lipoprotein binding of lipopolysaccharides in tolerant rabbit serum. J. Infect. Dis. 154:784-791 
37. Van Lenten, B. J., A. M. Fogelman, M. E. Haberland, and P. A. Edwards. 1986. The role of lipoproteins and receptor-mediated endocytosis in the transport of bacterial lipopolysaccharide. Proc. Natl. Acad. Sci. USA. 83:2704-2708.

38. Flegel, W. A., A. Wolpl, D. N. Mannel, and N. Northoff. 1989. Inhibition of endotoxin-induced activation of human monocytes by human lipoproteins. Infect. Immun. 57:2237-2245.

39. Cavaillon, J.-M., C. Fitting, N. Haeffner-Cavaillon, S. J. Kirsch, and H. S. Warren. 1990. Cytokine response by monocytes and macrophages to free and lipoprotein-bound lipopolysaccharide. Infect. Immun. 58:2375-2382.

40. Harris, H. W., C. Grunfeld, K. R. Feingold, and J. H. Rapp. 1990. Human very low density lipoproteins and chylomicrons can protect against endotoxin-induced death in mice. J. Clin. Invest. 86:696-702.

41. Frøholm, L. O., K. Jyssum, and K. Bøvre. 1973. Electron microscopical and cultural features of Neisseria meningitidis competence variants. Acta Pathol. Microbiol. Scand. Sect. B 81:525-537.

42. DeVoe, I. W., and J. E. Gilchrist. 1973. Release of endotoxin in the form of cell wall blebs during in vitro growth of Neisseria meningitidis. J. Exp. Med. 138:1156-1167.

43. Andersen, B. M., F. Skjørten, and O. Solberg. 1979. Electron microscopial study of Neisseria meningitidis releasing various amounts of free endotoxin. Acto Pathol. Microbiol. Scand. Sect. B 87:109-115.

44. Caugant, D. A., L. O. Frøholm, K. Bøvre, E. Holten, C. E. Frasch, L. F. Mocca, W. D. Zollinger, and R. K. Selander. 1986. Intercontinal spread of a genetically distinctive complex of clones of Neisseria meningitidis causing epidemic disease. Proc. Natl. Acad. Sci. USA. 83:4927-4931.

45. Westphal, O., O. Lüderitz, and F. Bister. 1952. Über die Extraktion von Bakterien mit Phenol/Wasser. Z. Naturforsch. 7B:148-155.

46. Galanos, C., O. Lüderitz, and O. Westphal. 1969. A new method for the extraction of R lipopolysaccharides. Eur. J. Biochem. 9:245-249.

47. Takayama, K., N. Qureshi, K. Hyver, J. Honovich, R. J. Cotter, P. Mascagni, and H. Schneider. 1986. Characterization of a structural series of lipid A obtained from the lipopoly saccharides of Neisseria gonorrhoeae. J. Biol. Chem. 261:10624-10631.
48. Bryn, K., A. Sonesson, R. Reistad, and E. Jantzen. 1988. Chemical characterization of lipopolysaccharide of Neisseria meningitidis 44/76. XIV International Carbohydrate Symposium, Stockholm, Sweden. Abstr. A77.

49. Wilkinson, S. G. 1988. Gram-negative bacteria. In Microbial Lipids, Volume 1. C. Ratledge and S. G. Wilkinson, editors. Academic Press, Ltd., London. 299-488.

50. Bryn, K., and E. Jantzen. 1986. Quantification of 2-keto-3-deoxyoctonate in (lipo)polysaccharides by methanolytic release, trifluoroacetylation, and capillary gas chromatography. J. Chromatogr. 370:103-112.

51. Schneider, H., T. L. Hale, W. D. Zollinger, R. C. Seid, C. A. Hammack, and J. M. Griffiss. 1984. Heterogeneity of molecular size and antigenic expression within lipooligosaccharides of individual strains of Neisseria gonorrhoeae and Neisseria meningitidis. Infect. Immun. 45:544-549.

52. Tsai, C.-M., R. Boykins, and C. E. Frasch. 1983. Heterogeneity and variation among Neisseria meningitidis lipopolysaccharides. J. Bacteriol. 155:498504.

53. Griffiss, J. M., H. Schneider, R. E. Mandrell, R. Yamasaki, G. A. Jarvis, J. J. Kim, B. W. Gibson, R. Hamadeh, M. A. Apicella. 1988. Lipooligosaccharides: the principal glycolipids of the neisserial outer membrane. Rev. Infect. Dis. 10:S287-S295.

54. Sober, H. A., editor. 1970. Handbook of Biochemistry, 2nd edition. Chemical Rubber Company, Cleveland, Ohio, C 3-35.

55. Frederichsen, P., and P. Kierulf. 1979. A more accurate dye-binding method for the routine determination of serum albumin. Clin. Chem. 25:1180

56. Clauss, A. 1957. Gerinnungsphysiologische Schnellmethode zur Bestimmung des Fibrinogens. Acta Haematol. 17:237-246.

57. Hirsch, P. B., A. Howie, R. B. Nicholason, and D. W. Pashley. 1965. Periodic and ordered structures. In Electron Microscopy of Thin Crystals. Butterworths, London, 353-387.

58. Applications Data, Spinco DS-502. Beckman Instruments, Inc., Palo Alto, CA. 